\title{
Creating And Working With Control Charts In Excel
}

\author{
Paul F. Schikora, Indiana State University, USA
}

\begin{abstract}
With the growth in distance education offerings, instructors who now teach quantitative material via the web have been faced with many challenges. Foremost has been the need to develop appropriate methods for teaching such material to students who are not physically in the classroom. Methodologies that have traditionally been taught in a highly interactive mode in the classroom must now be presented effectively in a far more asynchronous environment. Tutorials and detailed handouts are one way to accomplish this.

We present a written tutorial for creating quality control charts using Excel. The tutorial guides students through the process of creating $X$-bar and $R$ charts in such a way as to reinforce the theoretical basis of quality control already taught. Students apply their knowledge in hands-on activity, learn how to improve Excel's default charts to create visually effective control charts, and learn to reuse/recycle their work to easily create additional charts for different sets of problem data.
\end{abstract}

Keywords: Control Charts; Spreadsheets; Distance Education; Tutorial

\section{INTRODUCTION}

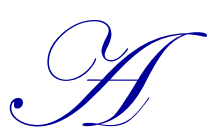

$s$ the growth of the Internet has led to a similar increase in the availability of courses to distance students, instructors who now teach quantitative material via the web have been faced with many challenges. Foremost has been the need to develop appropriate methods for teaching such material to students who are not physically in the classroom. For a many quantitative topics, traditional pedagogy has depended heavily on direct and interactive demonstrations in the classroom. In developing web-based courses, instructors have to face the fact that such direct, synchronous interaction is not possible. They must develop alternative, but effective methods to replace the classroom experience. This can be further complicated when the instructor has typically relied on specialized software, such as statistical packages, to teach certain topics. While this software may be readily available and fully supported on campus, distance students will often not have ready access to the specific package. When that is the case, not only does the instructor have to develop a distance-appropriate methodology, but that methodology must be built around a readily available software solution.

\section{Motivation}

We have faced this challenge in developing courses on statistical quality control for web delivery. Specifically, teaching the theory and development of quality control charts has, in the past, typically relied heavily on direct interactive demonstrations with students in a university computer lab. There, various statistical software packages can be demonstrated by the instructor, and the students can practice their new art with the guidance of the everpresent instructor. The teaching of the methodology can be done fairly quickly, with much of the learning coming hands-on as the students work on creating control charts from sample data. Student questions can be answered immediately, averting frustration on the students' parts, and quickly reinforcing the learning experience. In developing pedagogy for the new web courses, we realized we had been taking that direct interaction for granted.

We quickly realized that a new model for teaching control charts had to be developed. Without the interactive exercises, we realized that the web-based approach would have to be a more complete, detailed, and self-contained package. We would have to anticipate common student questions or uncertainty, and find alternative methods to 
somehow show what could not be directly demonstrated. We also could no longer rely on specialized software packages, such as Minitab or SPSS, because we could not expect all distance students to have such packages readily available for their use. So the new instructional methodology would have to include the use of a widely available software solution.

\section{Approach}

Research has shown that the availability of various learning resources in a web class is directly related to student performance in the class (Murray, et al., 2012). Rather than relying on any existing resource to bridge the distance gap, we decided to develop a tutorial to teach students how to develop control charts from a set of sample data. The tutorial was intended to directly replace classroom demonstration, so it was designed in such a way that it could lead a reasonably prepared student through the necessary steps to create (and revise if necessary) common control charts. When faced with the choice of an appropriate software package to do so, spreadsheets seemed an obvious choice. We developed the tutorial around the ubiquitous Microsoft Excel spreadsheet package, one to which most any student would have reasonably easy access. The exact method of creating charts in Excel varies based on the version of Excel being used - we use Excel 2013 in this tutorial.

The choice of Excel as a software package also presented us with a couple of side benefits. When demonstrating how to develop control charts in a typical statistical package, the student does not gain much experience in applying the underlying theory and methodology, as the statistical packages typically do all the work. The same is typically true even if using an Excel add-in to create the charts (e.g. Buttrey, 2009). They also tend to leave the student with little control over the final appearance of the control charts. The use of Excel addresses both those shortfalls. When building control charts in Excel, students are forced to work directly with sample statistics and control chart formulas, thus reinforcing earlier learning on the theoretical and statistical bases of control charts. Also, through the use of Excel's drawing tools, we could teach students a bit of the art of making good business presentations of their work. Both of these benefits add value for the student.

We had several primary goals for the tutorial:

- It should provide sufficiently detailed steps to lead an average student through the steps necessary to build control charts on his own, without necessarily being a complete technical manual.

- To minimize "busy work" on the part of the students, it should borrow some environmental concepts: reduce, reuse, and recycle. While developing control charts in Excel reinforces earlier learning, it can be a bit tedious to repeat the process from scratch for numerous example problem data sets. We wanted to present a method that would let students reapply their earlier work on later data sets.

- It should provide some ideas on how to format the charts to make them better visual tools for use in actual quality control situations.

Research has shown that the availability of various learning resources in a web class is directly related to student performance in the class (Murray, et al., 2012). While there are several resources available on using spreadsheets to build control charts (e.g. "Control Chart in Excel", n.d; "How to Create ...", n.d.), none sufficiently met our goals to be used as-is for our courses. There are also texts dedicated to quality control with spreadsheets [e.g. Zimmerman and Icenogle], but we desired ancillary material that would not add to the cost of taking the course and could be updated in-house for new releases of Excel.

\section{Tutorial}

The tutorial we developed has been a work in progress, and is currently fully functional as plug-and-play pedagogy for many courses. It has several features that have helped us accomplish the goals previously stated.

- The tutorial demonstrates all of the steps needed to take a set of sample data and create standard X-Bar and $\mathrm{R}$ charts. It assumes the student has a basic understanding of the theory, so focuses on the steps needed to develop the charts in Excel. Understanding of basic Excel concepts is assumed.

- Because direct demonstration is not possible, screen captures are used throughout to take the place of 
classroom demonstration. These images show the students how to perform certain steps, and also what they should see if they are proceeding correctly.

- It is written in an informal, conversational style. Feedback from our students indicate they prefer this approach over "technical manual" style writing.

- Tips on formatting the default chart to make it a good TQM visual tool are presented.

- Instructions on how to reuse already developed charts to create revised charts for the current data set, or new charts for additional data sets are provided. This is especially important in our courses, where the students must turn in both trial and revised control charts for homework and exam problems. Reusing charts can help cut down the tedium of working multiple problems.

- The tutorial can easily be supplemented by a screen-capture video recording with instructor voiceover if desired.

The tutorial, as used in our courses, is presented in the following section. It can be used as-is in most courses, or modified for specific needs. Comments and feedback are welcomed by the authors. 


\section{CREATING AND WORKING WITH CONTROL CHARTS IN EXCEL}

\section{Introduction}

There are many software packages that can create and update control charts for you. Some are specific to quality control, and most generalized statistical packages also have this capability. However, this course is not designed to train you in the use of any particular software program. Instead, the aim is to provide you with a solid understanding of the statistical basis for the construction and use of control charts. As such, you will be building and working with control charts from scratch, using Microsoft Excel or another similar spreadsheet program. This tutorial is designed to provide a reasonable approach to doing so for this class.

It is assumed that students have basic capabilities for working in Excel-meaning you know how to copy/paste data, use functions, select and manipulate cells, format how cells display, use absolute references, etc.

\section{Set It Up}

The first step of course is to enter the data you have collected (or have been given) into the spreadsheet -- we use the data in Appendix A. Enter your data in rows, one row for each sample. Each sample observation will be entered as a separate cell in the sample's row. Headings should also be used to help you keep track of what everything is. Parameters/factors necessary for creating the control chart need also be entered somewhere. We recommend you enter them below your sample data as shown in Figure 1 - the reason for this will become apparent later on. In this example, I have the control chart factors entered in cells B28 to B31. Finally, note that we have included columns for the sample statistics. (Figure 1). 
Figure 1. Initial Setup

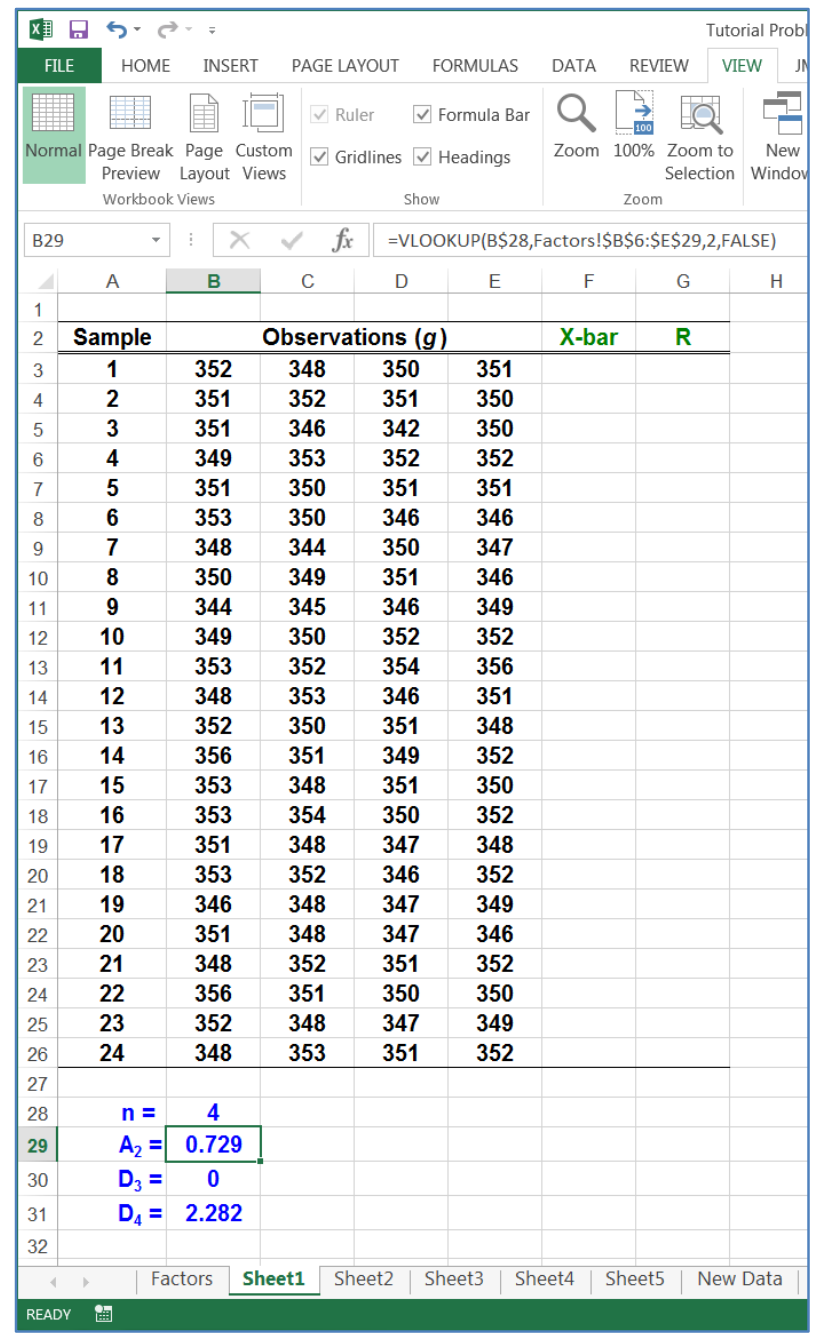

\section{Compute Sample Statistics}

For every control chart, you will compute sample statistics to be plotted on the control. These statistics should be computed on the same row as the relevant sample, using standard Excel formulas. In our example, X-bar is computed using the average () function, and the sample range ( $\mathrm{R}$ ) is computed using the $\max ()$ and $\min ($ ) functions. See the formula bar in the Figure 2 for an example of the latter.

To save time and effort, you should enter the formulas only once, and then copy and paste them into the other cells for the remaining samples.

\section{Compute Control Limits and Center Line}

The next step is to compute these key components of the control chart. We recommend computing them at the bottom of your sample data, again using standard Excel formulas. In computing these values, do not enter the values of any parameters/factors directly - rather, reference the cell that contains the value. For example (as can be seen in the formula bar in Figure 3), when computing the UCL for the X-bar chart, the value of factor $\mathrm{A}_{2}$ is not entered, but the cell containing its value, $\mathrm{C} 29$, is. Likewise, the value of X-double-bar is not entered, but rather cell 
F27 is, and so on. The reason for using cell references instead of direct values will become apparent when we look at modifying the control chart later on in this tutorial.

Figure 2. Sample Statistics

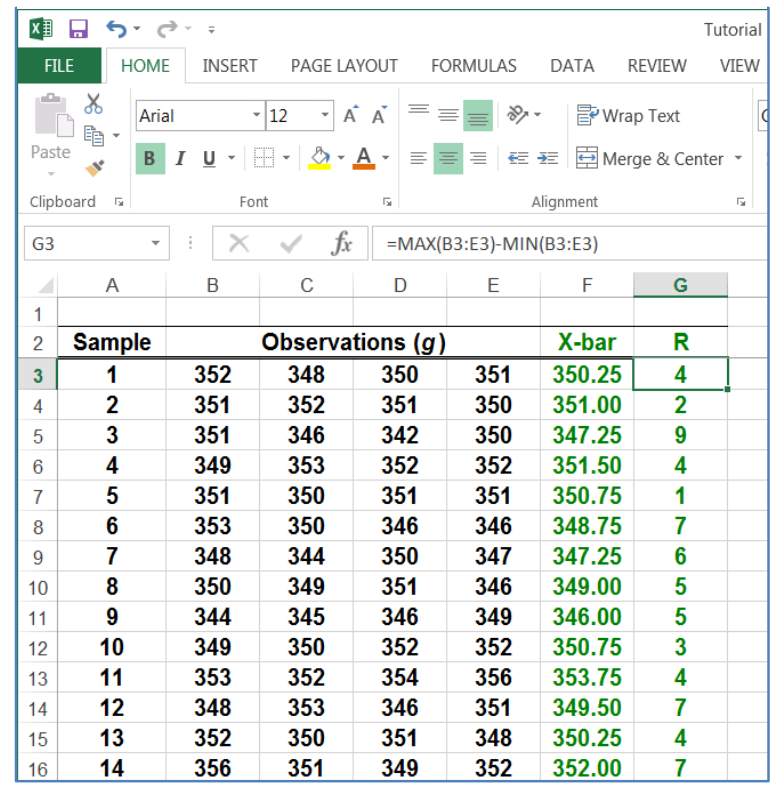

Figure 2. Calculating Center Line and Control Limits

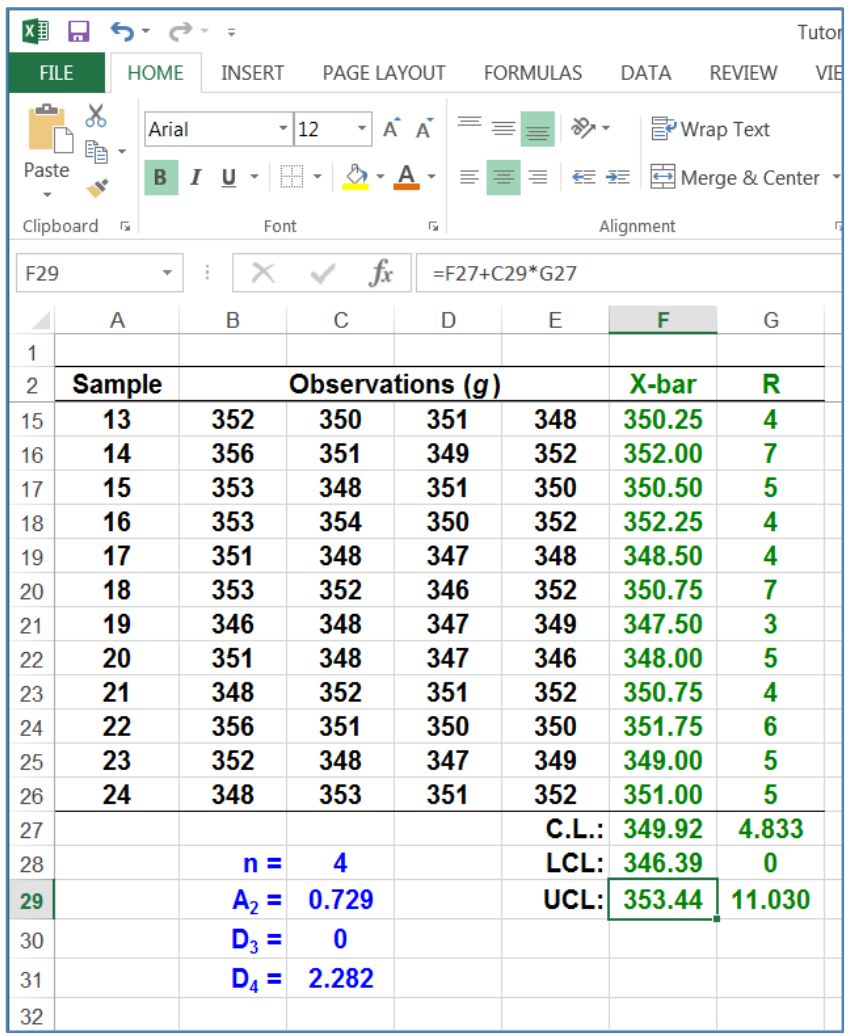

Create and Populate Columns for the Center Line and Control Limits: These horizontal lines can be manually drawn on a control chart, but it is much better to have Excel draw them. To do so, we'll create a column of identical numbers for each of these lines. To fill in the values, just make an absolute reference to the cell where you earlier computed the value for each line (the formula bar in Figure 4 gives an example - cell F27 contains the value of the center line for our chart). Do this for each of the control chart lines on the first row of sample data, and then copy the row to all the other rows. When these values are included in the control chart, they will be plotted as straight horizontal lines - just what we want! 
Figure 3. Columns for Center Line and Control Limits

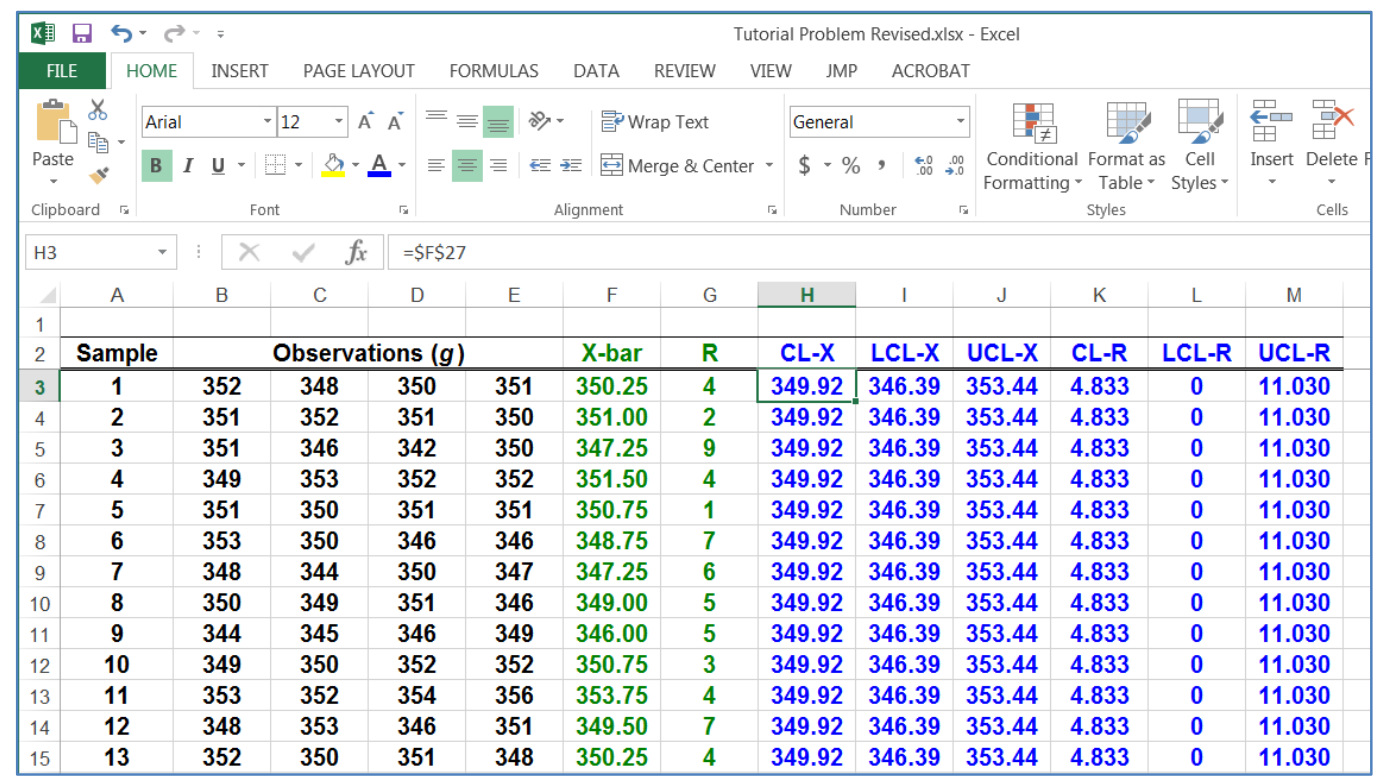

\section{Create the Charts}

At this point, the spreadsheet has all the data needed to draw the control chart. We will use the built-in charting capabilities of Excel to do this. Just follow a few steps, and your control chart will be created. The exact method depends on what version of Excel you are using. As throughout this tutorial we are using Excel 2013.

Highlight relevant cells.

Select (highlight) the cells containing the sample statistic of interest, the center line values, and the control limit values. A quick and easy way to do so is to hold down the $<$ Ctrl $>$ key and select the needed cells with the mouse. It is useful to include the column headers as well. See Figure 5. 
Figure 4. Selecting Chart Data

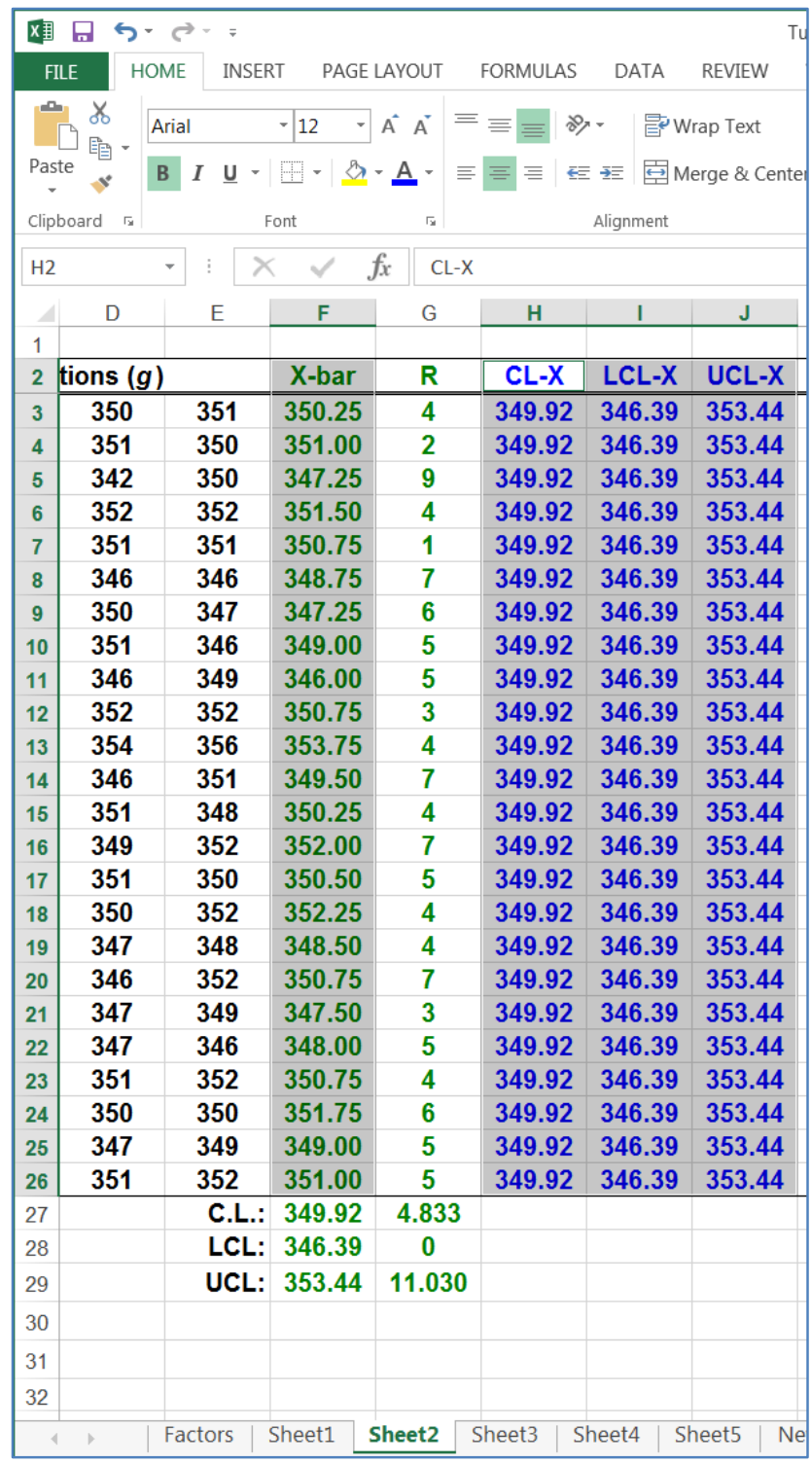

\section{Insert a Line Chart}

You can do this by navigating to the Insert tab, and in the Charts section of the ribbon click on the Insert Line Chart dropdown list and select the first option under 2-D Line (see Figure 6). As you hover your mouse over that option, a preview of the chart will show up - it should look like Figure 6. Click on that option and the chart will be inserted in your spreadsheet, as shown in Figure 7. 
Figure 5. Inserting Chart

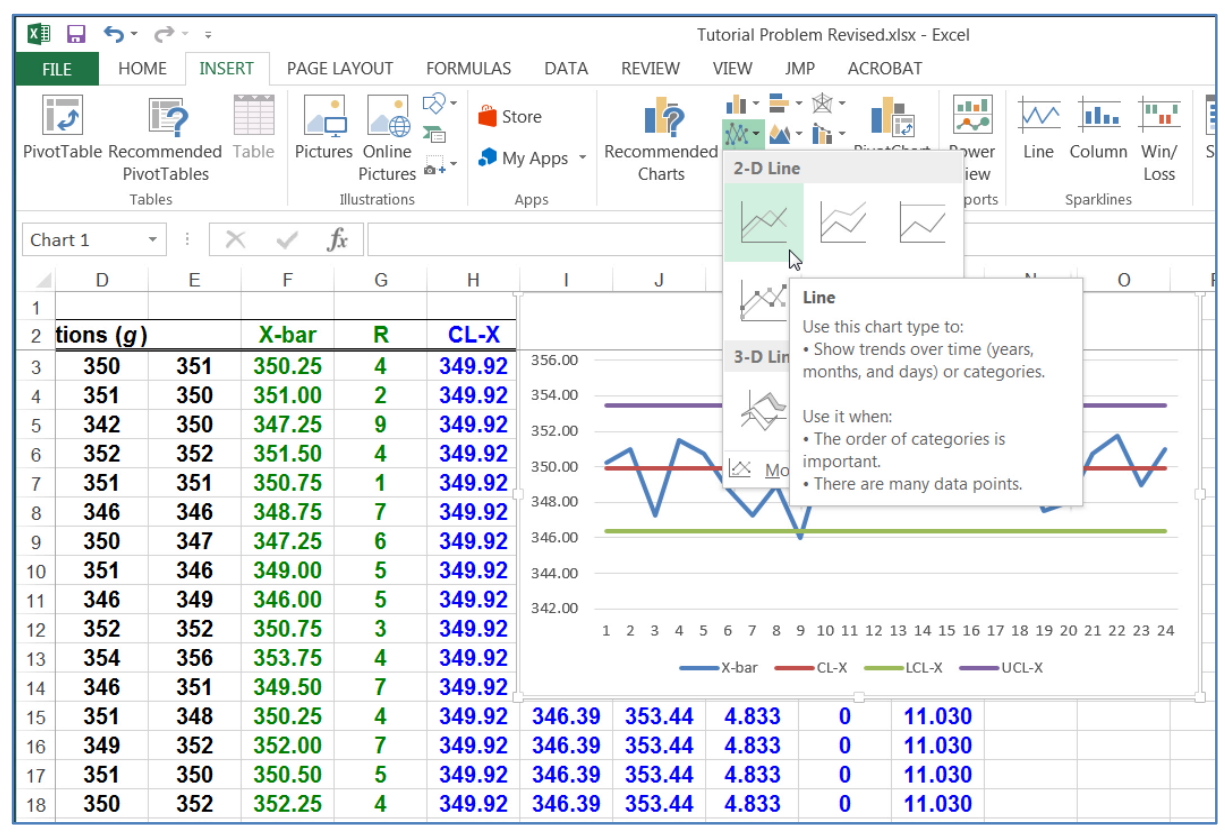

Figure 6. Initial Chart

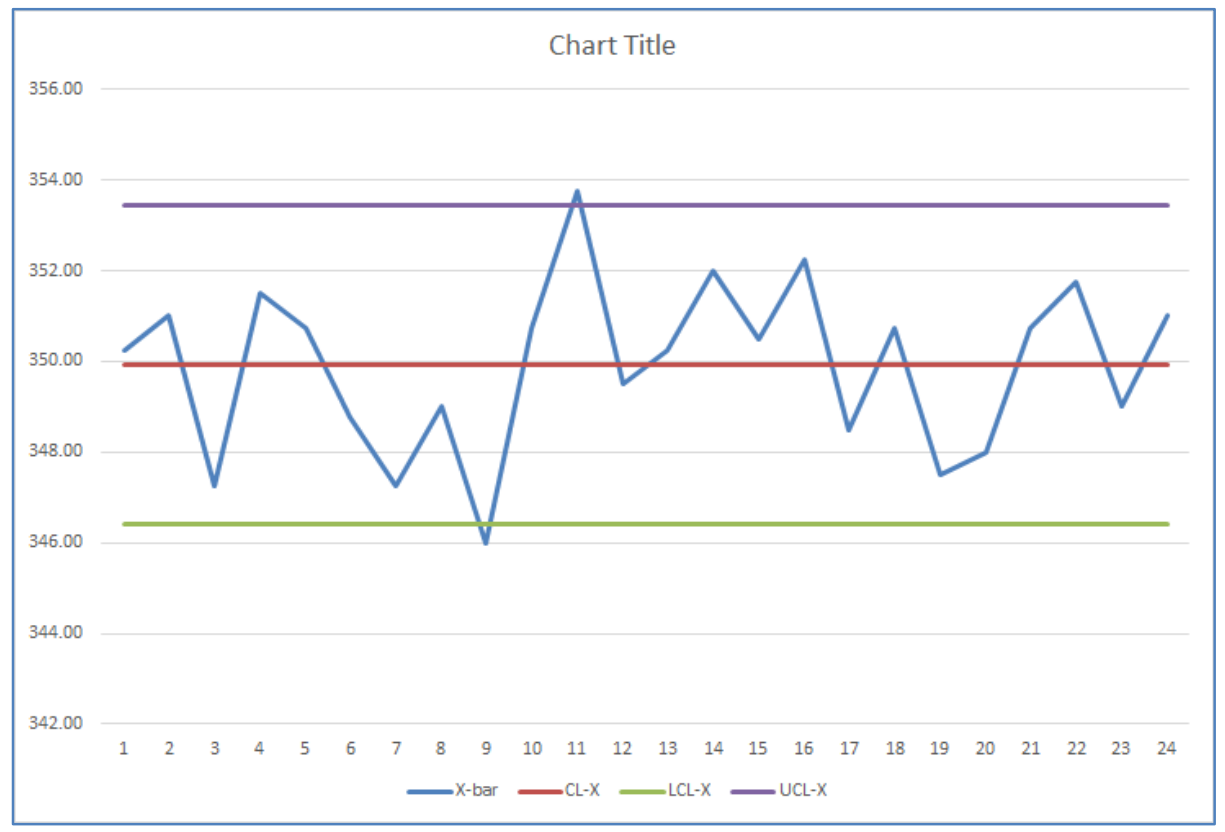

\section{Clean It Up}

When you are done, Excel has placed the chart into your spreadsheet, but the default formatting and placement will probably not be to your liking. All of the lines drawn on the chart will be different random colors, there will be no labels for the X-and Y-axes, and the chart title will be "Chart Title" by default. 


\section{Position It}

Click and drag the chart off to where you want it. You can drop the chart into blank space of the spreadsheet, or on top of your data.

Resize It

Change the size of the chart to your liking. Click on the chart, and then drag (left click, and move the mouse while holding the left mouse button) any of the 8 handles (small squares on the border of the chart) to resize the chart.

Change the Appearance

To make it look more like a typical control chart, there are a few formatting changes we recommend.

\section{$\underline{\text { Format the Run Line (Data Points) }}$}

If you have followed our example exactly, the run line of sample means will be medium blue, but will not have any markers to mark the data points. Whether or not it looks like our example, you may want to change how the run line appears. To do so, click the data line so that it is highlighted (each data point is now identified). Then, with the mouse cursor positioned on the line, right-click, and from the context menu that pops up, select Format Data Series (Figure 8). This will bring up the Task Pane titled Format Data Series on the right hand side of your worksheet. From within this task pane you can make many formatting changes to your chart. You can change the color and style of the line by clicking on the Fill $\&$ Lines icon (looks like a paint can pouring paint) shown under Series Options (Figure 9). You have further options to alter the line itself, or the markers to identify data points on the line. We are happy with the blue color for the line, but want to add markers to indicate each sample mean. To do so we select the Marker sub-option in the task pane, and under that Marker Options. By default None is selected. We choose Automatic to get round markers that match the color of the line. You can also choose the Built-in option to choose the shape and size of the marker if you prefer.

Figure 7. Formatting Run Line

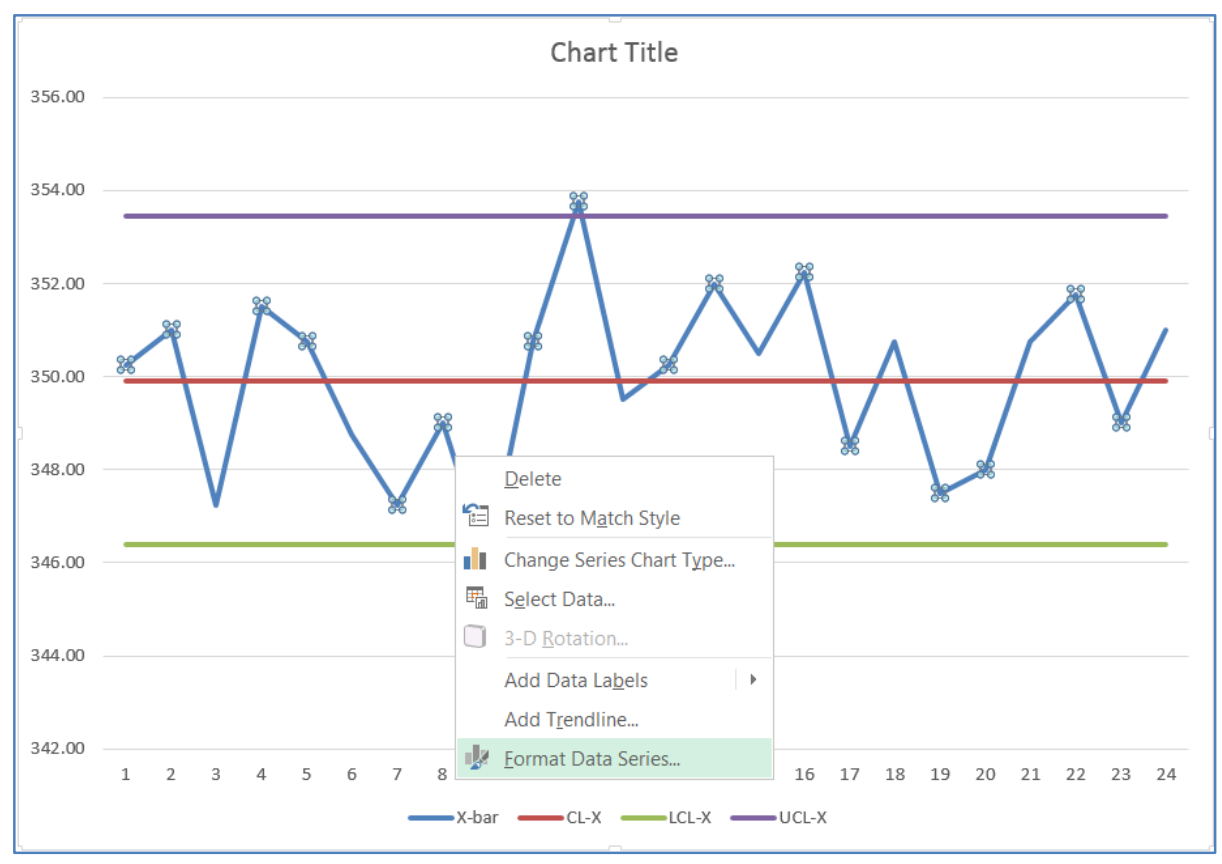


Figure 8. Formatting Options

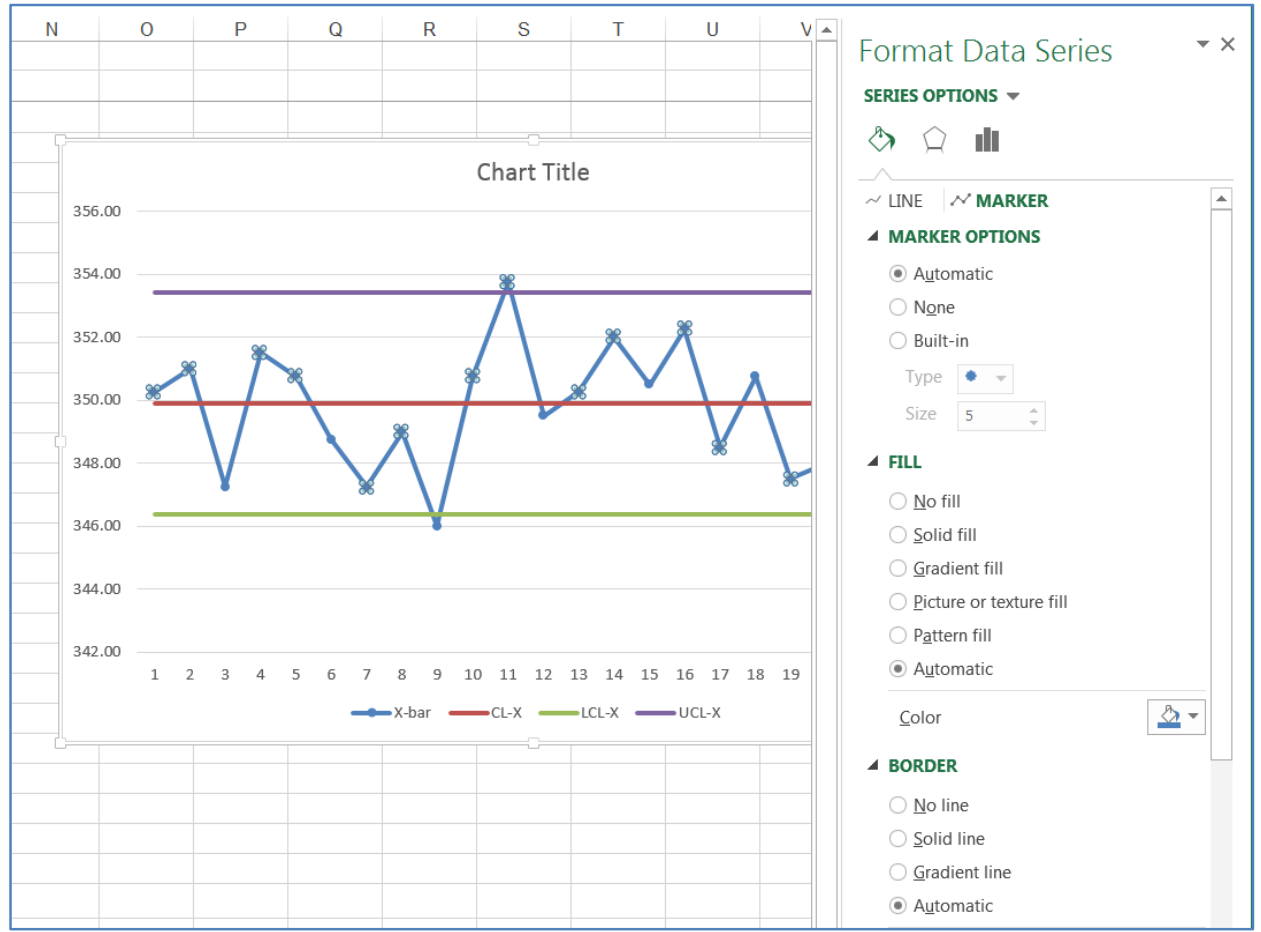

Figure 9. Selecting Other Lines

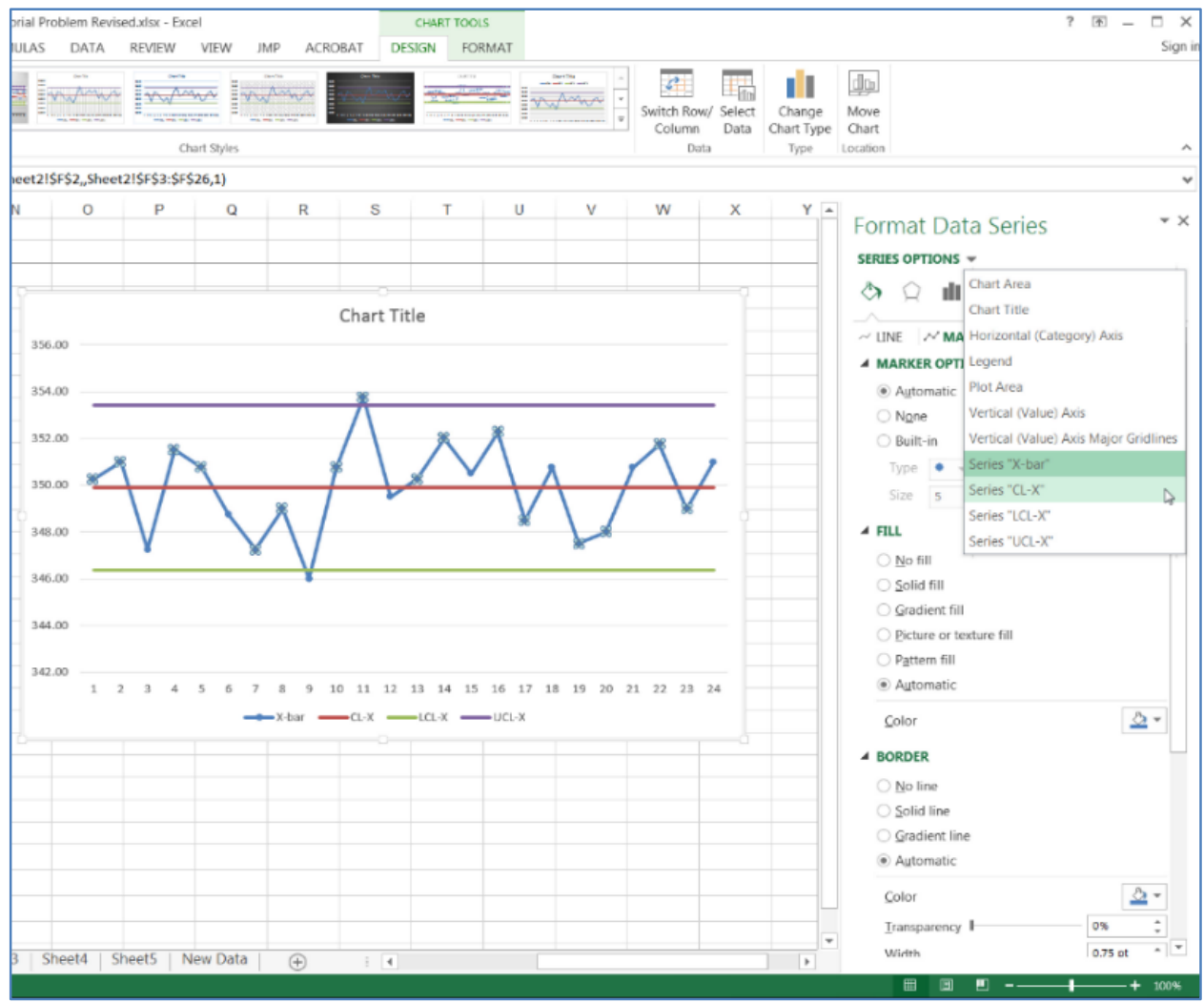




\section{Format Center Line and Control Limits}

At this point, each of these three lines is a different color, because Excel considers them three more data series, which have to be differentiated from each other. We know better though, and would like them to have a more consistent look, say a green center line, and red control limits. You reformat these lines, one at a time, in a manner similar to what you did for the data series line. Continuing to work in the Task Pane, select Series Options near the top which will give you a list of different parts of the chart you can format. Start by selecting the center line series so that you can reformat it (Figure 10). Then select the Line sub-option and change the color of the line to whatever you prefer. Repeat this process for the two control limits. When done you will have something that is starting to look more like a control chart (Figure 11).

Figure 10. Reformatted Lines

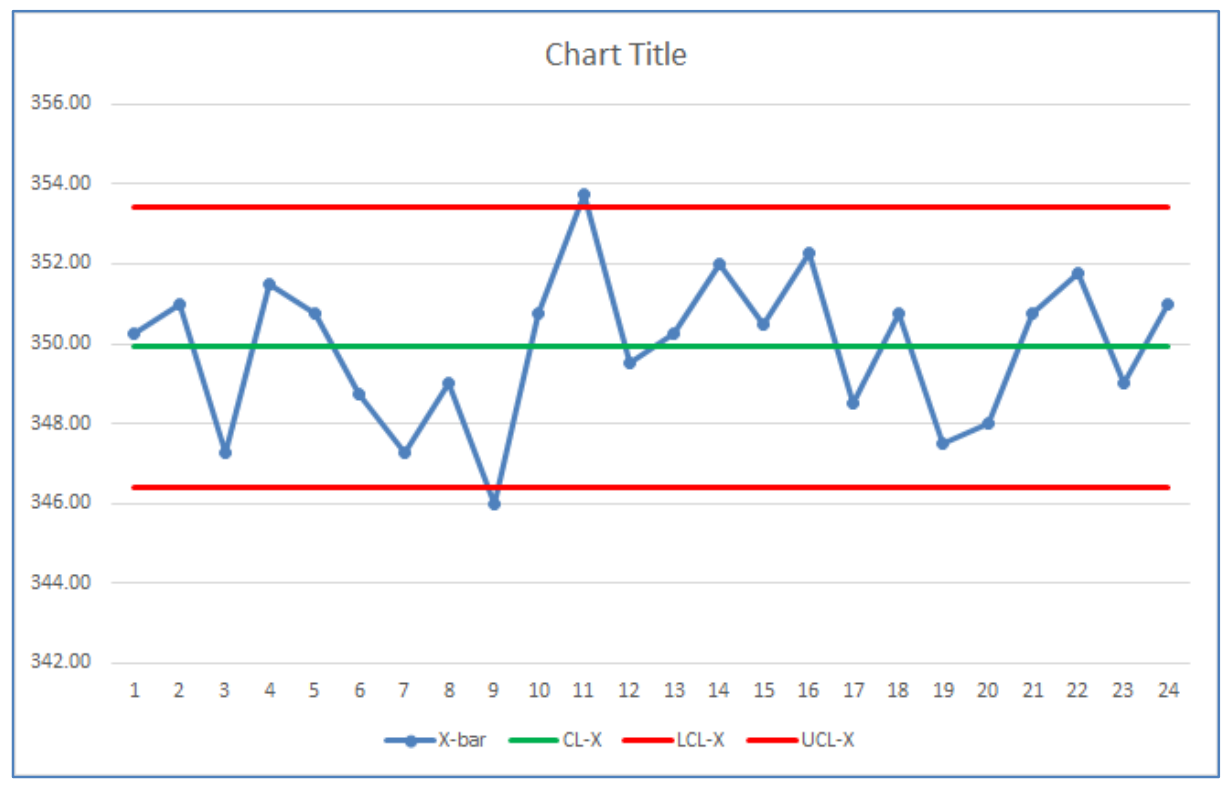

$\underline{\text { Change Title, Add Axis Labels }}$

You can change the title of your chart by simply clicking on in to set a cursor blinking in the title area. Delete the default and type in the title of your choice. We will need to add titles to the axes before we can edit them. Click anywhere in open space near the edge of your chart - you will see three icons appear to the upper right of the chart: a plus sign, a paintbrush, and a funnel. We want to add elements to the chart, so click on the plus sign icon. You will get a list of Chart Elements that you can add/delete from your chart (Figure 12). Currently included icons are shown by a checked box; missing elements have an empty box. To insert axis titles just check the box next to Axis Titles and you will see titles appear for both axes. Edit these just as you edited the chart title. Our finished chart is shown in Figure 13. 
Figure 11. Inserting Axis Titles

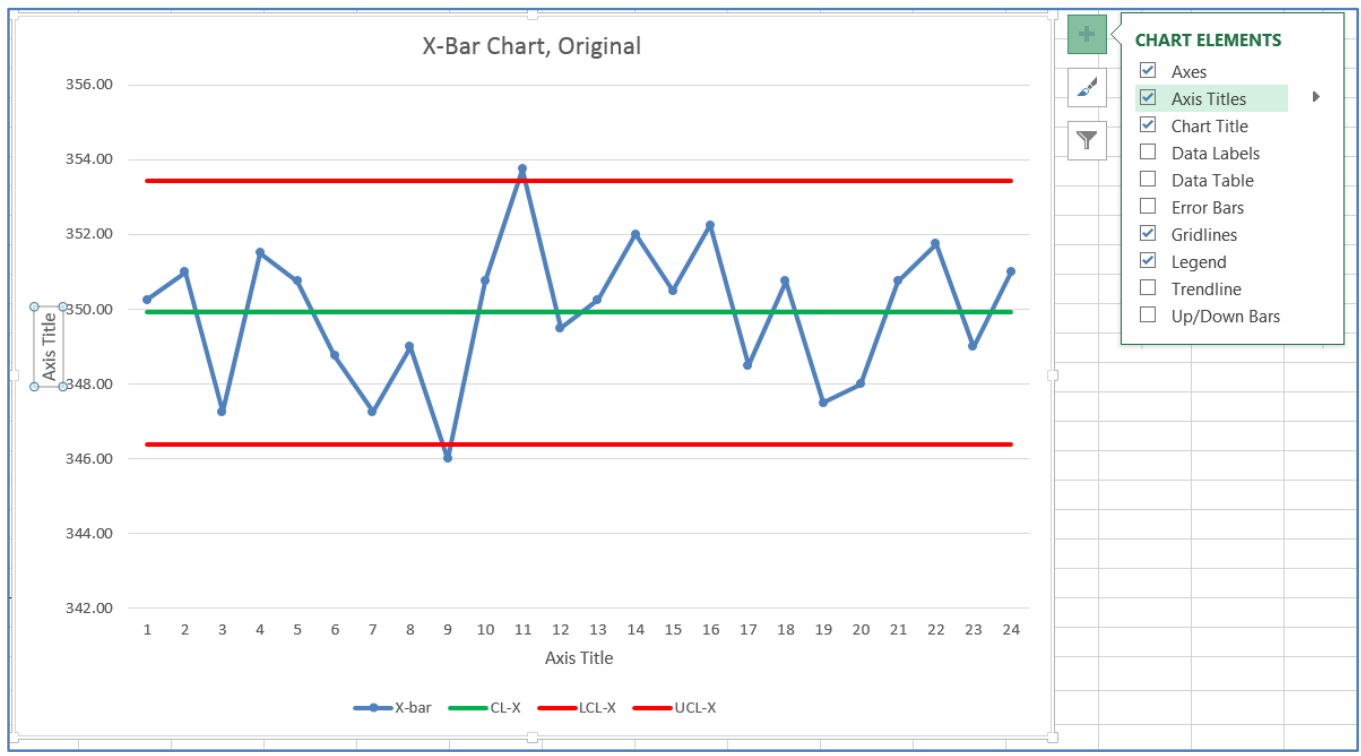

Figure 12. Final X-Bar Chart

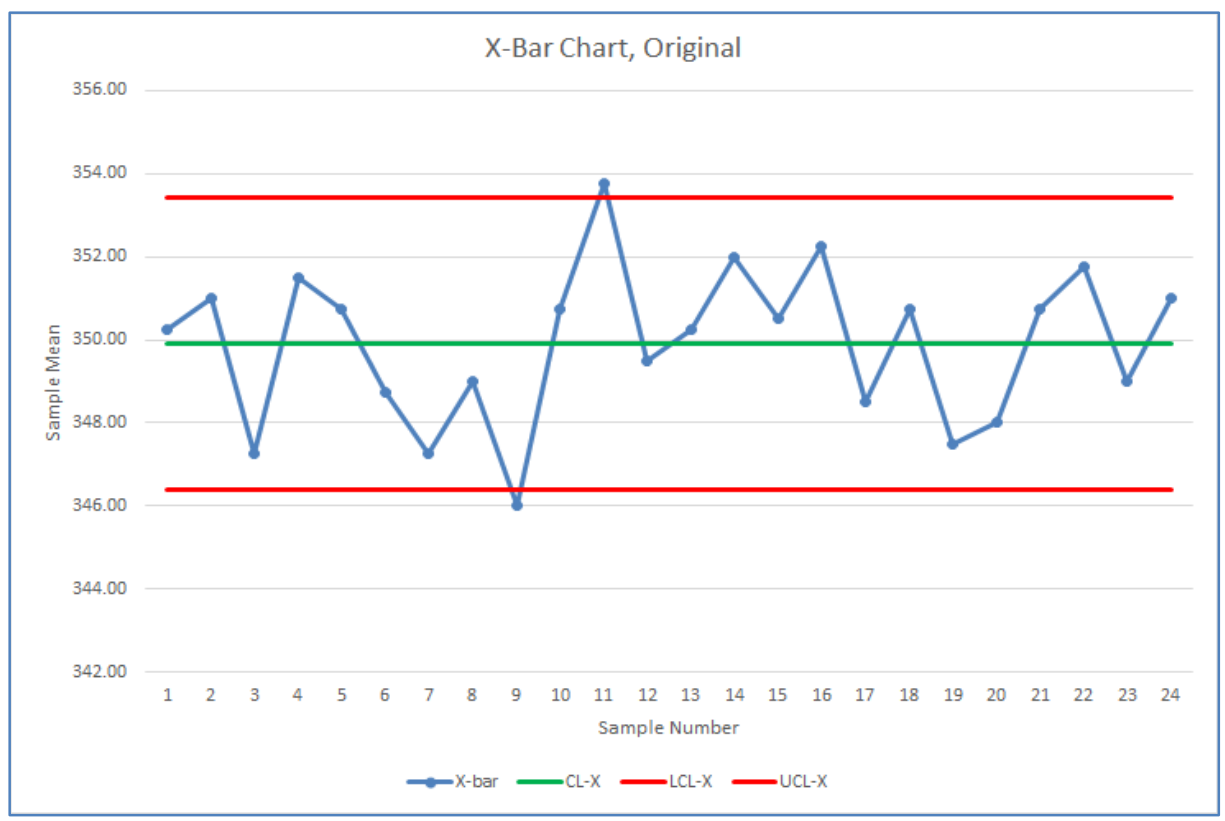

\section{Create Other Charts}

You can create any number of charts from the same data. For our purposes, we want to create an R-chart as well. You could repeat the process for creating the X-bar chart to create the R-chart. However, rather than starting from scratch and creating completely new charts, let's try and take advantage of the work we've already done. You can make a copy of the X-Bar chart you have just created, and modify it to become your R-Chart. Most of the formatting work you have already done will remain in this new chart, saving much work in creating your R-Chart. 
First, make a copy of your X-Bar Chart. Highlight the chart by clicking somewhere on the blank space in the chart, near the outer border. Then right click, and select Copy from the pop-up context menu. Right click on a blank cell in the spreadsheet, and select Paste. You will now have a duplicate of your X-Bar chart. At this point, update the chart title and axis labels for an R chart. You can now modify this copy to become your R chart by changing the source data for the chart. Right click somewhere on the blank space in the plot area of the chart and select Select Data from the context menu (Figure 14). You will be presented with a dialog window Select Data Source (Figure $15)$.

Figure 13. Changing Source Data

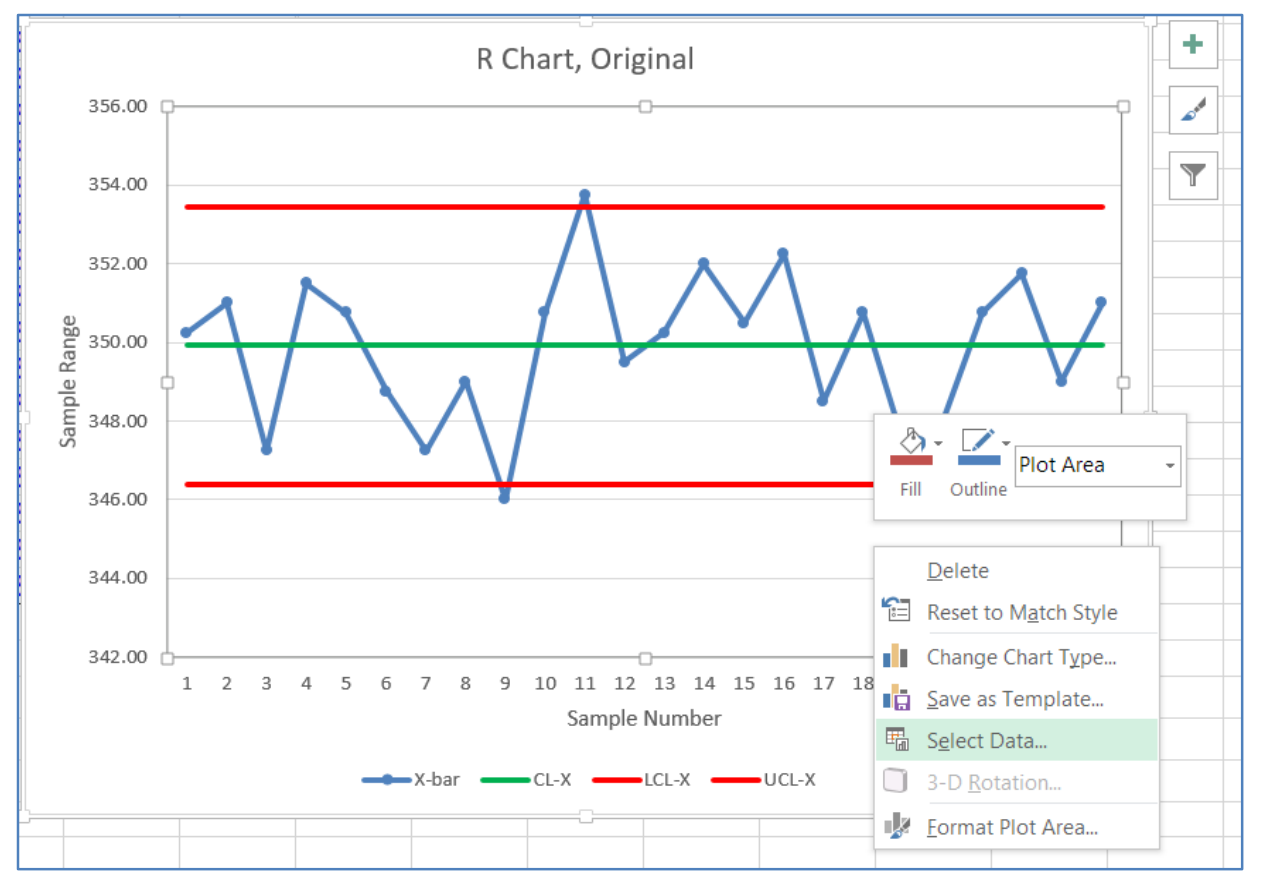

Figure 14. Select Source Data

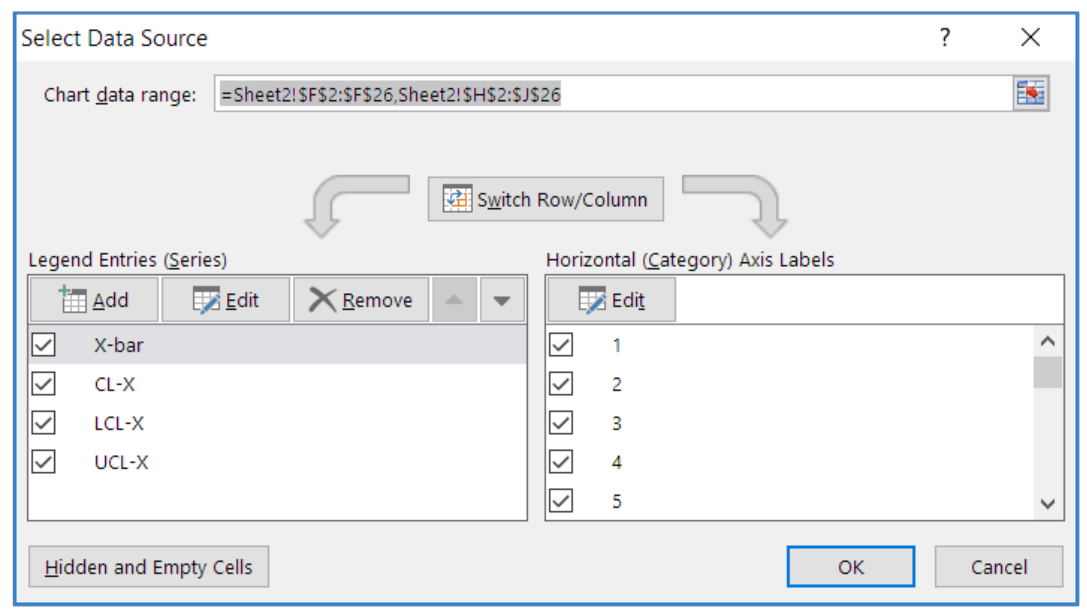

When the Select Data Source window is visible, you will notice that the data in the spreadsheet used to create your $\mathrm{X}$-Bar chart is outlined by moving dashed lines. This is the same data listed as the Chart Data Range in the Select Data Source window. You want to change the data cells associated with this chart to the data relevant for an $\mathrm{R}$ 
chart. Remember how you highlighted all the data cells to create the X-Bar chart in the first place (Figure 5)? You just need to repeat that process again, selecting the data cells associated with the $\mathrm{R}$ chart this time. Start by selecting the "R" column, and then using the $<\mathbf{C t r l}>$ key select the R center line and control limits columns. Once you start selecting those cells, you'll note that the chart updates automatically as you select the new data. You'll also notice though that you lose the formatting of the center line and control limits - that's OK ... you now know how to reformat them the way you want. When you have the $\mathrm{R}$ chart data selected on the spreadsheet, just click OK on the Select Data Source window, and your copy of the X-Bar chart will now be an R chart! Reformat any lines on the chart the way you want and you are done (Figure 16).

Figure 15. R Chart

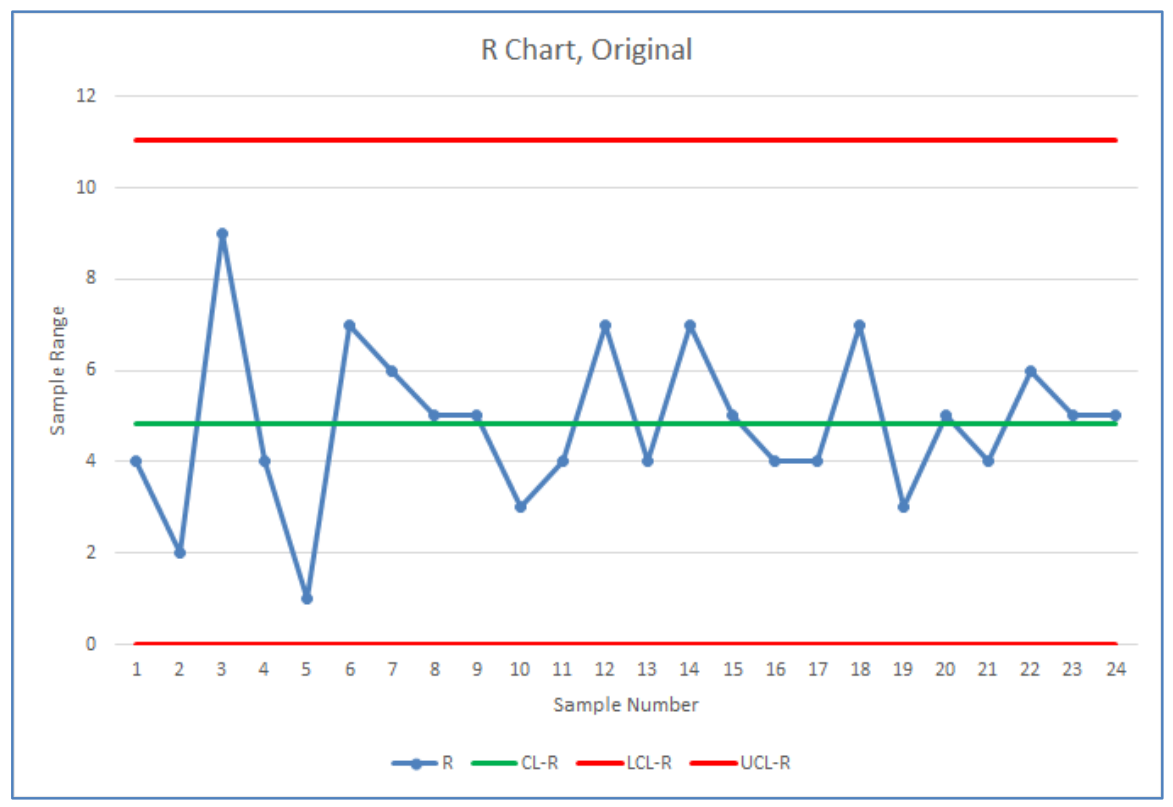

\section{Making Changes to the Control Chart}

Often, you will need to make changes to your control chart once you have determined that one or more of the initial data points need to be tossed out. Making changes is quite easy, because we used formulas and cell references in the creation of the original control chart.

\section{Change the Original or a Copy}

In the field, you would have no reason to maintain the original control chart, and can make changes to your original. In your coursework however, you will usually need to turn in copies of the original and the revised control charts for most assigned problems. When both are needed, you should make a copy of the original, and make changes to the copy. To make a copy, it is best to make a copy of the complete worksheet that the original control chart was created in. That way, all of the data and graphics can be duplicated and worked with in the new worksheet without modifying your original work. To create a copy of your existing worksheet, right click on the worksheet tab at the bottom of the screen, and select Move or Copy . . . (Figure 17). The resultant window (Figure 18) allows you to select where the worksheet goes, and whether you want a copy of the worksheet, or to simply move the original. Check the box next to Create a copy, and select which existing worksheet you want the new sheet inserted before. Usually, you will want it after the original chart, so you should select to insert the copy before the worksheet that comes directly after the existing chart. That sounds confusing, but is quite simple. In the example, the existing control chart is in the worksheet labeled "Original", and the worksheet after that is labeled "Sheet3" (Figure 17). To create a copy of "Original" and place it directly after itself, we would have to tell Excel to place it before "Sheet3" 
(Figure 18). Clicking the $\mathbf{O K}$ button at this point creates the copy of the original spreadsheet and gives it a unique name based on the original sheet's name - in this case, "Original (2)", indicating it is the second instance of "Original". You can rename the worksheet tabs to something more meaningful at any time - we'll call our new one "Revised".

Figure 16. Copy Worksheet

\begin{tabular}{|c|c|c|c|c|c|c|c|}
\hline 19 & 348.50 & 4 & 349.92 & 346.39 & 353.44 & 4.833 & 0 \\
\hline 20 & 350.75 & 7 & 349.92 & 346.39 & 353.44 & 4.833 & 0 \\
\hline 21 & 347.50 & 3 & 349.92 & 346.39 & 353.44 & 4.833 & 0 \\
\hline 22 & 348.00 & 5 & 349.92 & 346.39 & 35344 & 4833 & 0 \\
\hline 23 & 350.75 & 4 & 349.92 & 346.39 & Insert... & & 0 \\
\hline 24 & 351.75 & 6 & 349.92 & 346.39 & 园 Delete & & 0 \\
\hline 25 & 349.00 & 5 & 349.92 & 346.39 & Rename & & 0 \\
\hline 26 & 351.00 & 5 & 349.92 & 346.39 & \multirow{5}{*}{\multicolumn{2}{|c|}{$\begin{array}{l}\text { Move or Copy... } \\
\text { Q View Code } \\
\text { 娄 } \\
\text { Protect Sheet... } \\
\text { Iab Color }\end{array}$}} & 0 \\
\hline 27 & 349.92 & 4.833 & & & & & \\
\hline 28 & 346.39 & 0 & & & & & \\
\hline 29 & 353.44 & 11.030 & & & & & \\
\hline 30 & & & & & & & \\
\hline 31 & & & & & \multirow{2}{*}{\multicolumn{2}{|c|}{ Hide }} & \\
\hline 32 & & & & & & & \\
\hline s & p & Factors & Sheet1 & Original & \multicolumn{2}{|c|}{ Select All Sheets } & Sheet5 \\
\hline
\end{tabular}

Figure 17. Insert Copy

\begin{tabular}{|l|l|l|}
\hline Move or Copy & ? & $\times$ \\
Move selected sheets & & \\
Io book: & & \\
\hline Tutorial Problem Revised.xlsx & & \\
\hline Before sheet: & & \\
\hline $\begin{array}{l}\text { Factors } \\
\text { Data } \\
\text { Original }\end{array}$ & \\
\hline Sheet3 & & \\
\hline $\begin{array}{l}\text { Sheet4 } \\
\text { Sheet5 } \\
\text { New Data } \\
\text { (move to end) }\end{array}$ & & \\
\hline$\square$ Create a copy: & & \\
\hline & & \\
\hline
\end{tabular}

\section{Make the Changes}

At this point, you are ready to make changes to your original chart. Because you used formulas and cell references earlier, any changes you make to your data (changing observation values, deleting a sample or observation, etc.) will be immediately and correctly reflected in the control chart. This would not be possible had you copied or directly entered simple numeric values. 
In our example, samples 9 and 11 fall outside the control limits on the X-Bar chart (Figure 13). Assuming causes were identified for these samples, the sample data should be discarded, and a revised control chart be created. This is a simple task given the way we have created the control chart. We need to delete the sample data for samples 9 and 11. However, we cannot simply delete the data in the cells for those samples - this would leave blank areas in our control chart with no data. Rather, we must delete the entire spreadsheet row associated with each of these samples. To delete a spreadsheet row, first highlight the row by clicking on the row number at the far left of the spreadsheet. To highlight more than one row, hold down the $<$ Ctrl $>$ key and click on additional row numbers. The rows you have selected will be clearly highlighted. In the example, rows 11 and 13 contain samples 9 and 11 , respectively, so these rows are selected. (A common mistake would be to select rows 9 and 11, instead of samples 9 and 11. Be careful to avoid that mistake.) To delete the rows, right-click on one of the highlighted rows, and select Delete from the pop-up context menu (Figure 19).

Figure 18. Deleting Data Rows

\begin{tabular}{|c|c|c|c|c|c|c|c|}
\hline 10 & 8 & 350 & 349 & & $351 \quad 340$ & 349.00 & 5 \\
\hline 11 & 9 & 344 & 345 & \multicolumn{3}{|c|}{ Xo cut } & 5 \\
\hline 12 & 10 & 349 & 350 & \multirow{2}{*}{\multicolumn{3}{|c|}{ 舅 Copy }} & 3 \\
\hline 13 & 11 & 353 & 352 & & & & 4 \\
\hline 14 & 12 & 348 & 353 & \multicolumn{3}{|c|}{ Paste Options: } & 7 \\
\hline 15 & 13 & 352 & 350 & \multicolumn{3}{|c|}{ 表 } & 4 \\
\hline 16 & 14 & 356 & 351 & \multicolumn{3}{|c|}{ Paste Special... } & 7 \\
\hline 17 & 15 & 353 & 348 & \multicolumn{3}{|c|}{ Insert } & 5 \\
\hline 18 & 16 & 353 & 354 & \multirow{2}{*}{\multicolumn{3}{|c|}{ Delete }} & 4 \\
\hline 19 & 17 & 351 & 348 & \multirow{2}{*}{\multicolumn{3}{|c|}{ Clear Contents }} & 4 \\
\hline 20 & 18 & 353 & 352 & & & & 7 \\
\hline
\end{tabular}

When you have deleted these rows from the revised sheet, you will notice that the control chart on the revised sheet has been automatically updated. This is because Excel is capable of adjusting formulas when entire rows or columns are deleted from or added to a spreadsheet. Because we used formulas to compute the center line and control limits, and we used formulas to reference these values in creating the lines for the chart, all of these values, and therefore the chart, are updated automatically when we delete a row. If you have created several charts from the same data, all of the charts will be updated at the same time. Using this tactic of copying worksheets before revising control charts can save you a lot of time on your assignments.

\section{Reuse and Recycle Your Charts}

Coming up with revised control charts is not the only use for a copy of a control chart worksheet. You can also take a worksheet developed for one set of data, and modify it to create the same type of control charts for an entirely different set of data. This again is thanks to the fact that we used formulas and references in the original spreadsheet. As you add, delete, or completely replace data in the sheet, the control charts automatically revise themselves to reflect those changes.

Let's say you are done with the problem used in this example, and want to now create charts for another set of data with 30 samples of 5 observations each (Appendix B). Our current worksheet only has 24 samples of 4 observations each. However, you can insert rows to make space for the extra samples, and likewise, you can insert a column to make room for the extra observation in each sample. If you do it correctly, the control charts will be updated to reflect the new data.

Start by making another copy of our original worksheet and working within this new worksheet. First, let's add the six rows needed to make room for 30 samples. To do this, highlight the entire spreadsheet rows associated with the 
last six samples. Then right-click somewhere in the highlighted area and select Insert from the context menu (Figure 20).

Figure 19. Inserting Rows

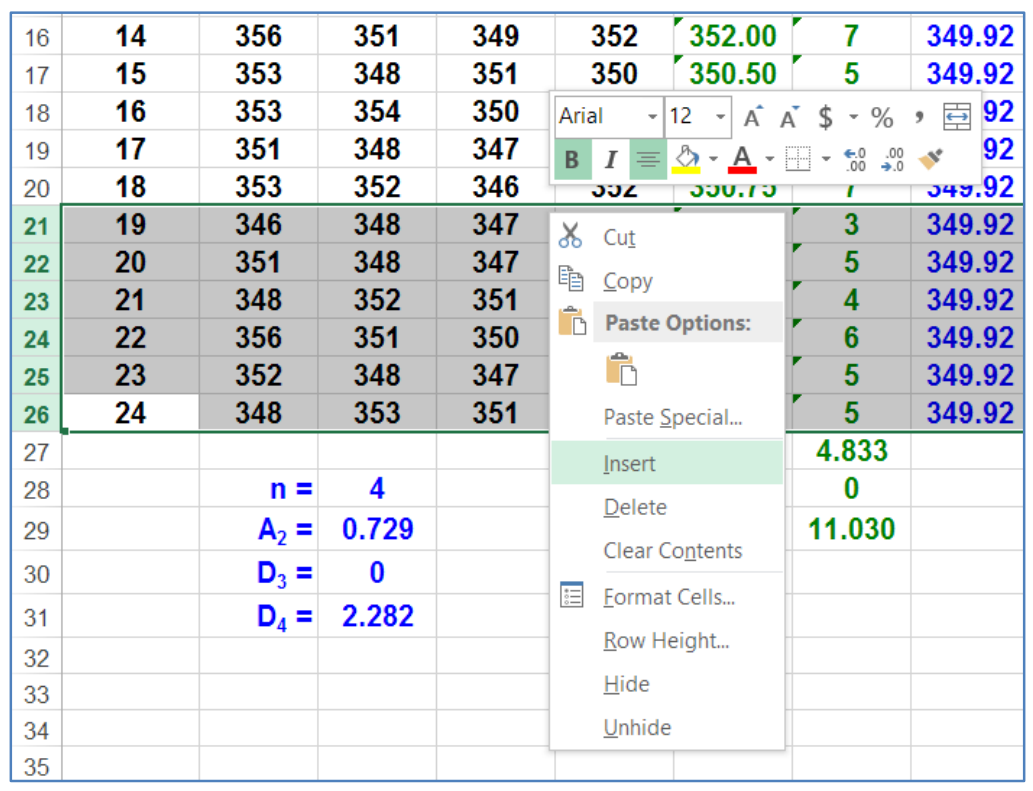

Once you have done this, six new, empty rows will be inserted in the data table. To convince yourself that the control chart has adjusted to the change, take a look at your charts. You will see that they have widened to adjust for the added rows, but there is a gap in the chart because we have not added data to the rows we inserted. Once you put data in those blank cells, the control chart will get updated automatically.

Similarly, you would insert a column by highlighting the last column of data (click on the column letter at the top of the spreadsheet), right-clicking, and selecting Insert from the context menu. You'll now have a blank column in the data table, waiting for data to be inserted. At this point, you would replace the existing data with your new data.

You will also need to do some cleanup work:

- Renumber the samples $(1,2,3, \ldots, 30)$ because we inserted the new rows into the middle of the old rows.

- Copy the formulas for the sample statistics, control limits, and center lines (columns G-N in the example spreadsheet) into the newly inserted rows.

- $\quad$ Change the control chart factors $\mathrm{A}_{2}, \mathrm{D}_{3}$, and $\mathrm{D}_{4}$, because the sample size $n$ changed from 4 to 5 .

- Adjust chart formatting if needed to make the new charts more to your liking.

When completed you'll have a new control chart(s) based on the new data. Using the data in Appendix B, our new results are shown in Figure 21. 
Figure 20. Control Charts for New Data Set

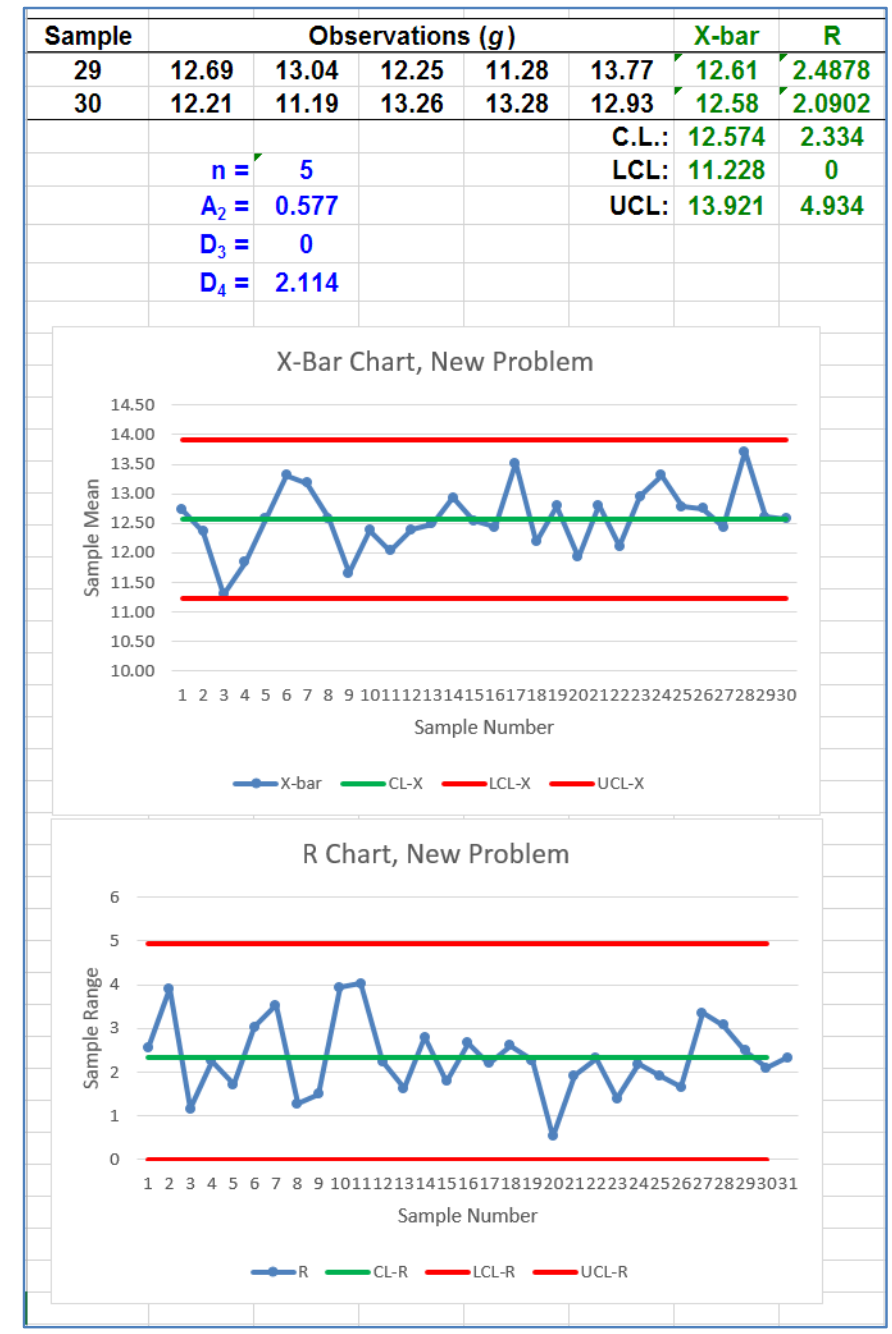

\section{AUTHOR BIOGRAPHY}

Dr. Schikora is Professor of Operations \& Supply Chain Management in the Scott College of Business at Indiana State University. He earned his Ph.D. in Operations Management from Indiana University's Kelley School of Business.

\section{REFERENCES}

Buttrey, S. E. (2009). An Excel Add-In for Statistical Process Control Charts. Journal of Statistical Software, 30(13), 1-12.

Control Chart Template. (n.d.) Retrieved from: http:/www.vertex42.com/ExcelTemplates/control-chart.html/

Control Chart in Excel - Create Six Sigma Quality Control Chart Using Excel. (n.d.) Retrieved from: http://www.databison.com/control-chart-in-excel/

How to Create a Control Chart. (n.d.) Retrieved from: http://www.wikihow.com/Create-a-Control-Chart/

Murray, M., et al. (2012). Student Interaction with Online Course Content: Build It and They Might Come. Journal of Information Technology Education: Research, 11, 125-140.

Zimmerman, S. M. \& Icenogle, M. L. (2003). Statistical Quality Control Using Excel 2/e, ASQ Quality Press. 


\section{APPENDIX A}

\section{Initial Control Chart Data}

\begin{tabular}{|c|c|c|c|c|}
\hline \multirow{2}{*}{$\frac{\text { Sample }}{1}$} & \multicolumn{4}{|c|}{ Observations } \\
\hline & 352 & 348 & 350 & 351 \\
\hline 2 & 351 & 352 & 351 & 350 \\
\hline 3 & 351 & 346 & 342 & 350 \\
\hline 4 & 349 & 353 & 352 & 352 \\
\hline 5 & 351 & 350 & 351 & 351 \\
\hline 6 & 353 & 350 & 346 & 346 \\
\hline 8 & 350 & 349 & 351 & 346 \\
\hline 9 & 344 & 345 & 346 & 349 \\
\hline 10 & 349 & 350 & 352 & 352 \\
\hline 11 & 353 & 352 & 354 & 356 \\
\hline 12 & 348 & 353 & 346 & 351 \\
\hline 13 & 352 & 350 & 351 & 348 \\
\hline 14 & 356 & 351 & 349 & 352 \\
\hline 16 & 353 & 354 & 350 & 352 \\
\hline 17 & 351 & 348 & 347 & 348 \\
\hline 18 & 353 & 352 & 346 & 352 \\
\hline 19 & 346 & 348 & 347 & 349 \\
\hline 20 & 351 & 348 & 347 & 346 \\
\hline 21 & 348 & 352 & 351 & 352 \\
\hline 22 & 356 & 351 & 350 & 350 \\
\hline 23 & 352 & 348 & 347 & 349 \\
\hline 24 & 348 & 353 & 351 & 352 \\
\hline
\end{tabular}




\section{APPENDIX B}

\section{Additional Control Chart Data}

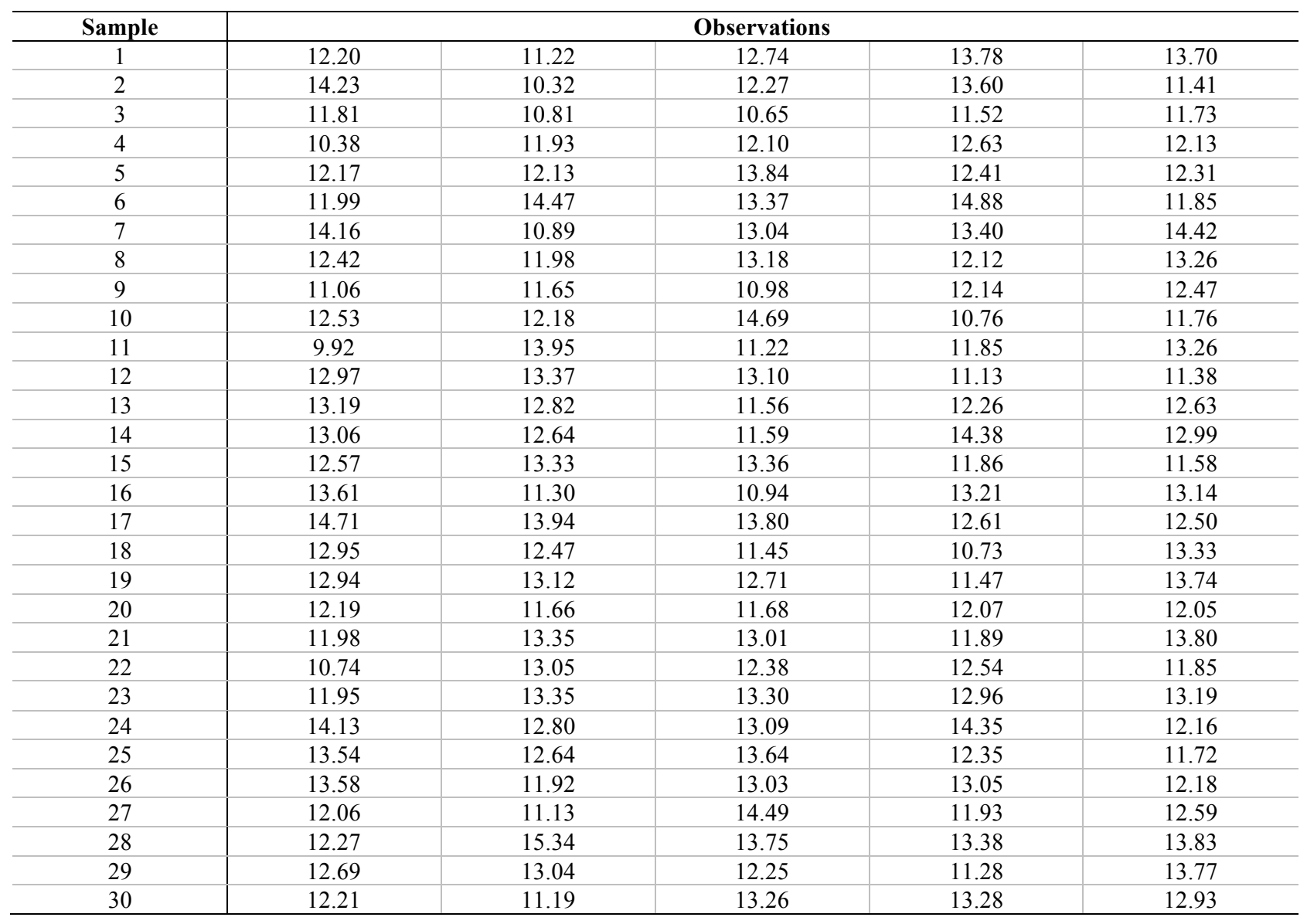




\section{NOTES}

